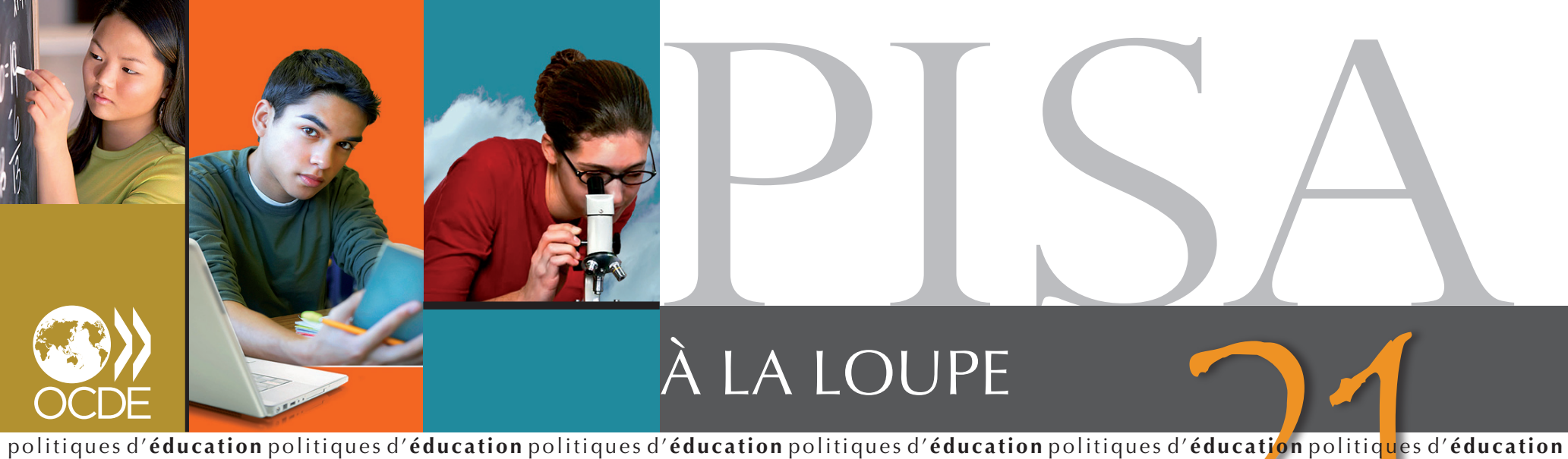

\title{
Aujourd'hui, les jeunes de 15 ans se sentent-ils responsables à l'égard de l'environnement?
}

- Dans les pays de l'OCDE, la plupart des jeunes de 15 ans ont une certaine connaissance des problèmes environnementaux et estiment que les menaces environnementales constituent un grave sujet d'inquiétude pour eux-mêmes et/ou pour d'autres personnes dans leur pays.

- La compréhension scientifique de l'environnement est essentielle pour que les élèves aient une vision réaliste des défis environnementaux que l'humanité doit relever. Les élèves dont les connaissances en sciences sont insuffisantes sous-estiment systématiquement le temps nécessaire pour résoudre des problèmes environnementaux tels que le traitement des déchets nucléaires ou l'extinction de certaines espèces animales et végétales.

La connaissance et la compréhension scientifique de l'environnement peuvent permettre d'éveiller chez les élèves un certain sens des responsabilités à l'égard de l'environnement. Toutefois, si les jeunes ne parviennent pas à établir un lien entre ce qu'ils apprennent à l'école et les défis environnementaux qui les entourent dans la vie réelle, ou s'ils font preuve d'un optimisme ou d'un pessimisme démesurés quant à la possibilité de relever ces défis, ils peuvent alors ne pas être en mesure de tirer pleinement parti de leur apprentissage scolaire sur ces sujets. L'école - et les parents - peuvent aider les élèves à établir ce lien et à adopter des attitudes réalistes pour relever ces défis.

Compréhension L'enquête PISA 2006 a évalué la compréhension scientifique des élèves et responsabilisation faces aux problèmes environnementaux et les a interrogés - ainsi que vont de pair. leurs parents dans certains pays - sur leurs attitudes à l'égard de ces problèmes. Les élèves devaient indiquer s'ils étaient au courant de problèmes tels que la pollution de l'air, les pénuries d'énergie, l'extinction de certaines espèces animales et végétales, l'abattage des forêts en vue de l'exploitation des sols, les pénuries d'eau et les déchets nucléaires. En outre, les élèves et leurs parents devaient indiquer si ces problèmes constituaient un grave sujet d'inquiétude pour eux-mêmes ou pour d'autres personnes dans leur pays, et s'ils étaient optimistes quant à la possibilité de trouver des solutions pour améliorer la situation au cours des 20 prochaines années.

La grande majorité des élèves de 15 ans ont indiqué être au courant des problèmes environnementaux ou avoir reçu un enseignement à ce sujet. En moyenne, dans les pays de l'OCDE, moins de $3 \%$ des élèves ont indiqué n'être pas sûrs de savoir ce qu'est «la pollution de l'air » ou "l'extinction de certaines espèces animales et végétales ». Cette proportion s'établit à seulement $5 \%$ environ concernant « les pénuries d'eau » et " l'abattage des forêts en vue de l'exploitation des sols "; à moins de $10 \%$ concernant "les pénuries d'énergie »; et enfin, à 11 \% concernant « les déchets nucléaires». 
Ce que les élèves apprennent en classe influe sur leurs attitudes en dehors de l'école

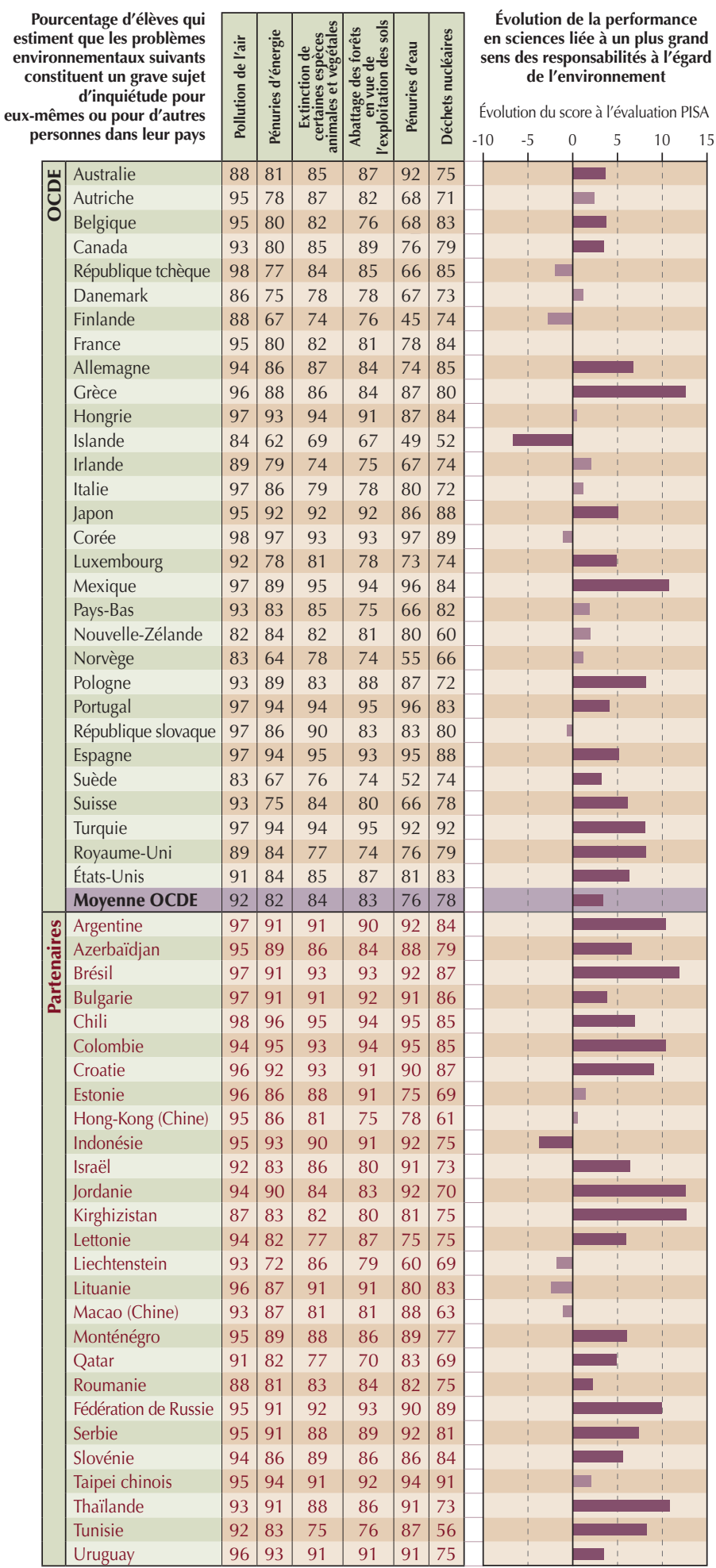

Remarque : les évolutions statistiquement significatives de la performance en sciences sont indiquées en couleur plus foncée. L'évolution de la performance en sciences correspon à l'évolution du score à l'évaluation PISA des compétences en sciences associée à la variation d'une unité de l'indice de responsabilisation des élèves à l'égard des problèmes environnementaux, après contrôle du milieu des élèves et des établissements.

PISA and Geoscience in PISA 2006, tableau A3.14.

StatLink तiाs $\mathrm{http}: / / \mathrm{dx} . \mathrm{doi} .0 \mathrm{rg} / 10.1787 / 562200685357$

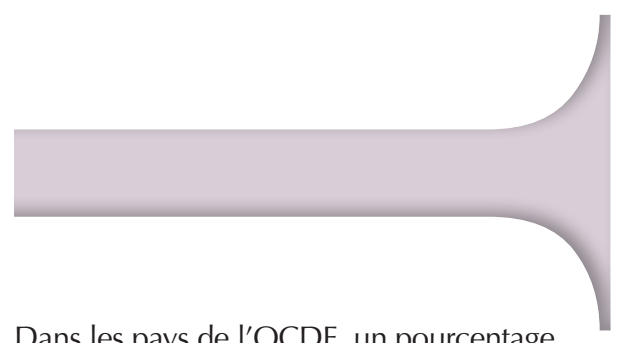

Dans les pays de l'OCDE, un pourcentage significatif d'élèves font part d'un sens aigu des responsabilités individuelles et collectives à l'égard des problèmes environnementaux - c'est-à-dire qu'ils indiquent que ces problèmes environnementaux constituent un grave sujet d'inquiétude pour eux-mêmes et/ou pour d'autres personnes dans leur pays. Ainsi, en moyenne, dans les pays de l'OCDE, $92 \%$ des élèves considèrent que la pollution de l'air constitue un grave sujet d'inquiétude pour eux-mêmes ou pour d'autres personnes dans leur pays. Cette proportion s'établit à plus de $80 \%$ concernant les pénuries d'énergie, l'extinction de certaines espèces animales ou végétales, et l'abattage des forêts ; et enfin, à environ $78 \%$ et $76 \%$, respectivement, concernant les pénuries d'eau et les déchets nucléaires.

\section{Mais l'optimisme quant à l'avenir} de l'environnement se fait rare..

Les élèves ne se montrent pas optimistes quant à la possibilité d'une atténuation significative des menaces environnementales au cours des 20 prochaines années. Ainsi, en moyenne, dans les pays de l'OCDE, seuls $15 \%$ des élèves, voire moins, estiment qu'il y aura une amélioration concernant les problèmes de déchets nucléaires, d'extinction de certaines espèces animales et végétales, et d'abattage des forêts en vue de l'exploitation des sols. Cette proportion s'établit à $16 \%$ concernant la pollution de l'air ; à seulement $18 \%$ concernant la lutte contre les pénuries d'eau ; et enfin, à $21 \%$ concernant les pénuries d'énergie.

L'école semble jouer un rôle central en tant que vecteur de connaissances sur les problèmes environnementaux. Les jeunes de 15 ans qui ont pris part à l'enquête PISA indiquent ainsi que leur principale source d'information sur l'environnement est l'école. 


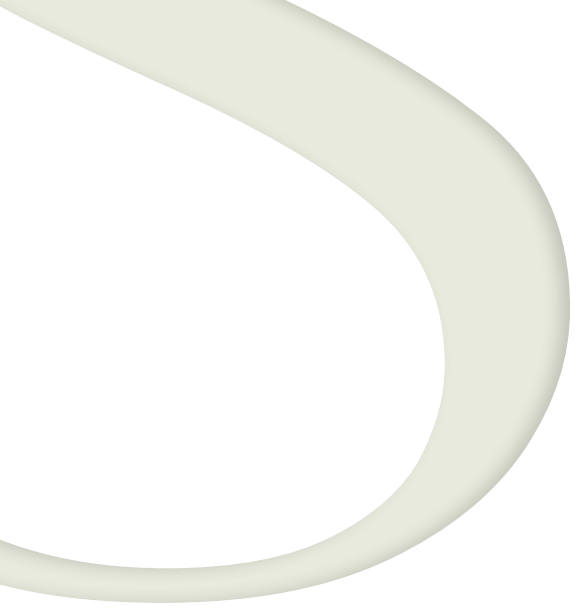

En moyenne, dans les pays de l'OCDE, $58 \%$ des élèves déclarent que l'école est leur principale source d'information concernant les problèmes de déchets nucléaires. Cette proportion s'établit à $59 \%$ concernant les pénuries d'eau; à environ $60 \%$ concernant les pénuries d'énergie ; à $65 \%$ concernant I'abattage des forêts en vue de l'exploitation des sols ; à $70 \%$ concernant l'extinction de certaines espèces animales et végétales; et enfin, à 76 \% concernant la pollution de l'air.

Si les élèves acquièrent des connaissances sur l'environnement à l'école, les familles jouent également un rôle essentiel dans la formation de leurs attitudes et de leurs opinions à l'égard des problèmes environnementaux, rôle qui, à son tour, peut influer sur leur volonté d'adopter ultérieurement un comportement respectueux de l'environnement. Les élèves partagent souvent le sens des responsabilités et l'optimisme de leurs parents à l'égard de l'environnement, bien que l'intensité de cette corrélation varie selon les pays et s'avère plus forte pour l'optimisme que pour le sens de responsabilités individuelles. À titre d'exemple, en Turquie et, dans les pays partenaires, en Colombie, les élèves et leurs parents ont répondu de façon identique à la question de savoir si les problèmes environnementaux allaient s'atténuer au cours des 20 prochaines années, tandis que la similitude de leurs réponses n'était pas aussi évidente en Allemagne, en Corée, au Danemark, en Islande, au Luxembourg et en Nouvelle-Zélande. En Turquie et, dans les pays partenaires, en Colombie, les élèves et leurs parents s'accordent aussi généralement à considérer que la plupart de ces problèmes environnementaux constituent un grave sujet d'inquiétude pour eux-mêmes et/ou pour d'autres personnes dans leur pays ; au Danemark, en Islande et au Luxembourg, en revanche, les élèves et leurs parents ont souvent donné des réponses différentes à ce sujet.

L'environnement : ça vous rend optimistes ?

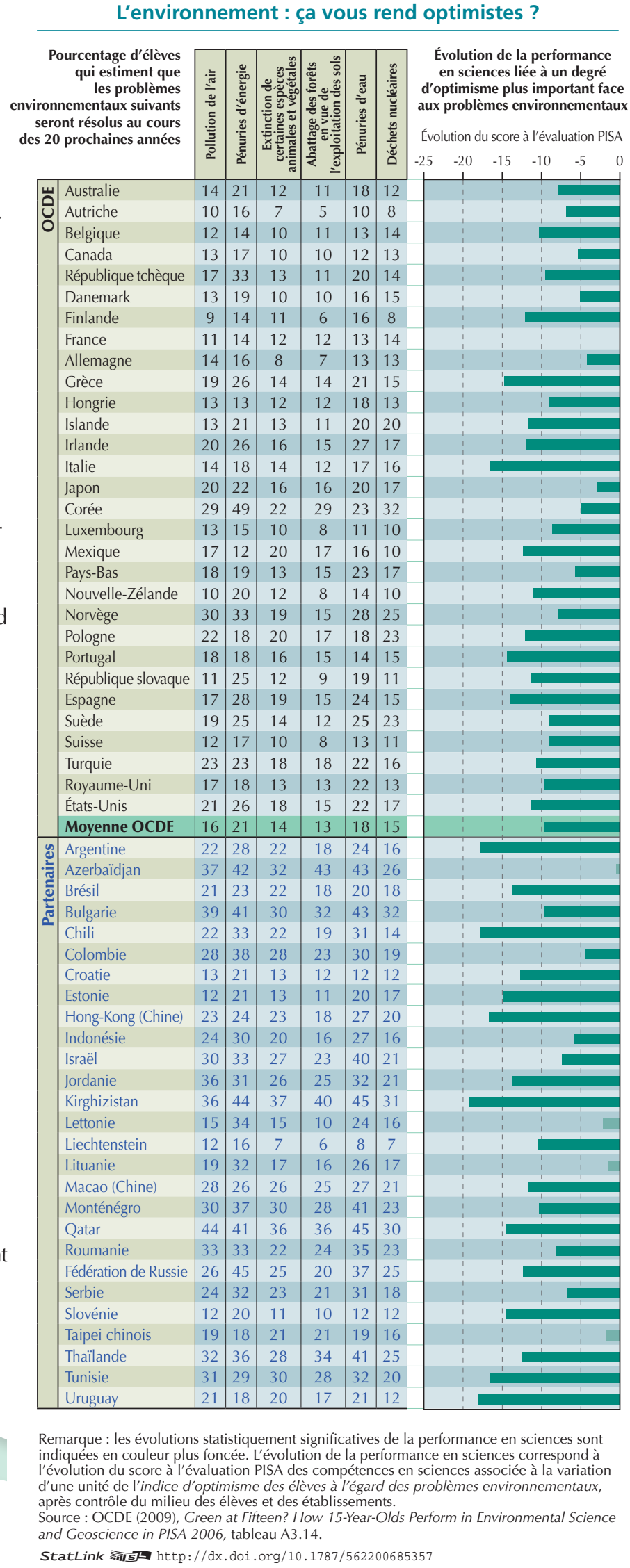

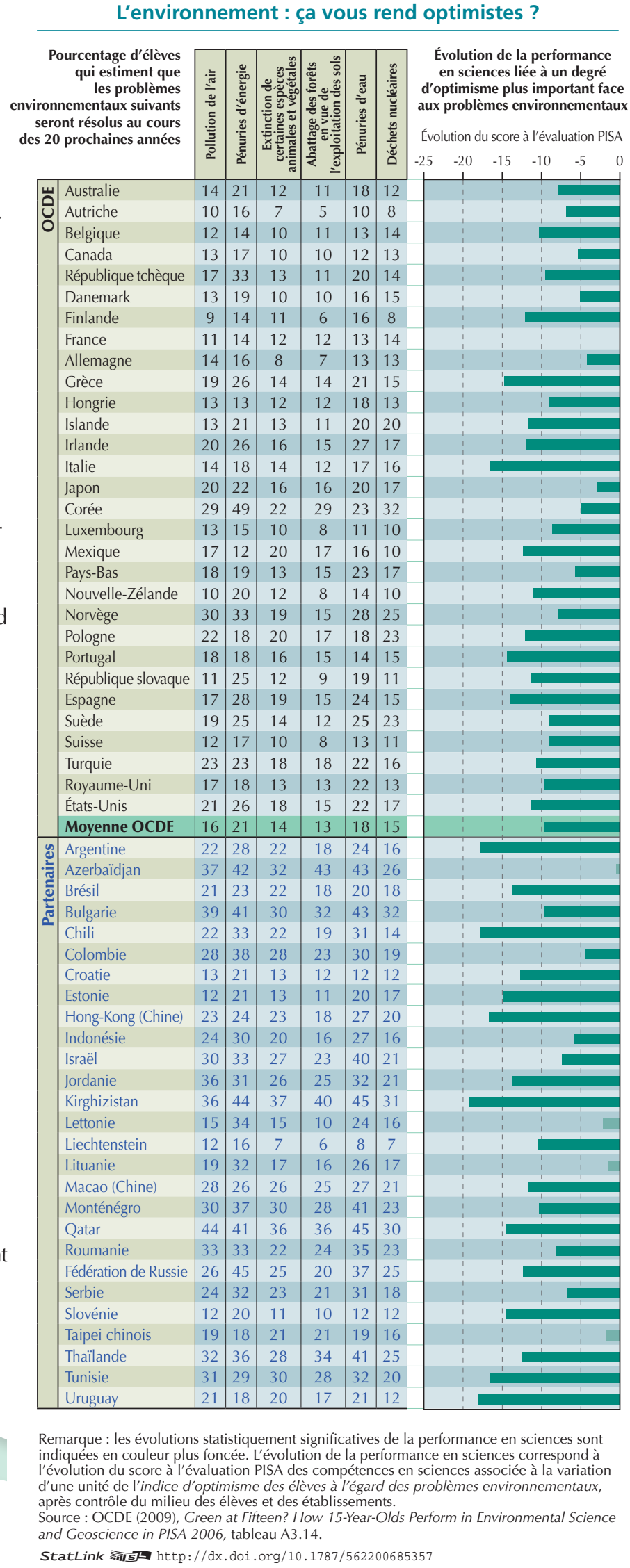

Remarque : les évolutions statistiquement significatives de la performance en sciences sont indiquées en couleur plus foncée. L'évolution de la performance en sciences correspond à l'évolution du score à l'évaluation PISA des compétences en sciences associee à la variation d'une unité de l'indice d'optimisme des élèves à 'egard des problèmes environnementaux après controle du milieu des éleves et des établissements. $P$

15-Year-Olds Perform in Environmental Science and Geoscience in PISA 2006, tableau A3.14.

StatLink त्ञाज $\mathrm{http}: / / \mathrm{dx}$.doi.org $/ 10.1787 / 562200685357$

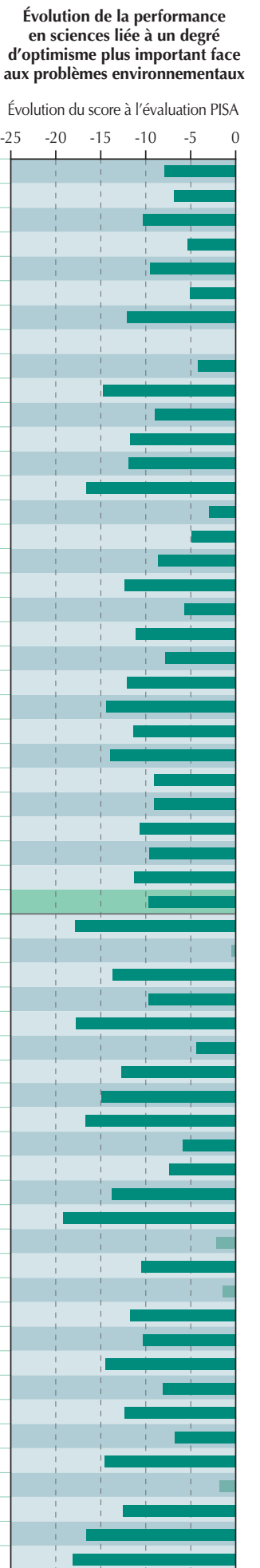

\section{À LA LOUPE}

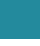


... et s'avère souvent lié à de moins bons résultats en sciences de l'environnement.

Selon les résultats de l'enquête PISA 2006, lorsque I'on compare des élèves issus de milieux et d'établissements d'enseignement similaires, il n'existe pas de relation évidente entre la performance des élèves en sciences de l'environnement et leur sens des responsabilités à l'égard de l'environnement. En revanche, il existe une corrélation négative entre le degré d'optimisme des élèves quant à la possibilité de résoudre les problèmes environnementaux au cours des 20 prochaines années et leur performance en sciences de l'environnement : plus les résultats des élèves en sciences de I'environnement sont faibles, plus ils se montrent optimistes quant à l'évolution des problèmes environnementaux au cours des 20 prochaines années. Cette tendance peut s'expliquer par le fait que les élèves qui n'ont pas une bonne compréhension des problèmes environnementaux sont dès lors susceptibles de se montrer plus optimistes, ou encore que les élèves qui sont plus optimistes quant à l'avenir de l'environnement sont moins enclins à approfondir leurs connaissances en sciences de l'environnement.

Les élèves partagent largement le sens des responsabilités et l'optimisme de leurs parents face aux problèmes environnementaux

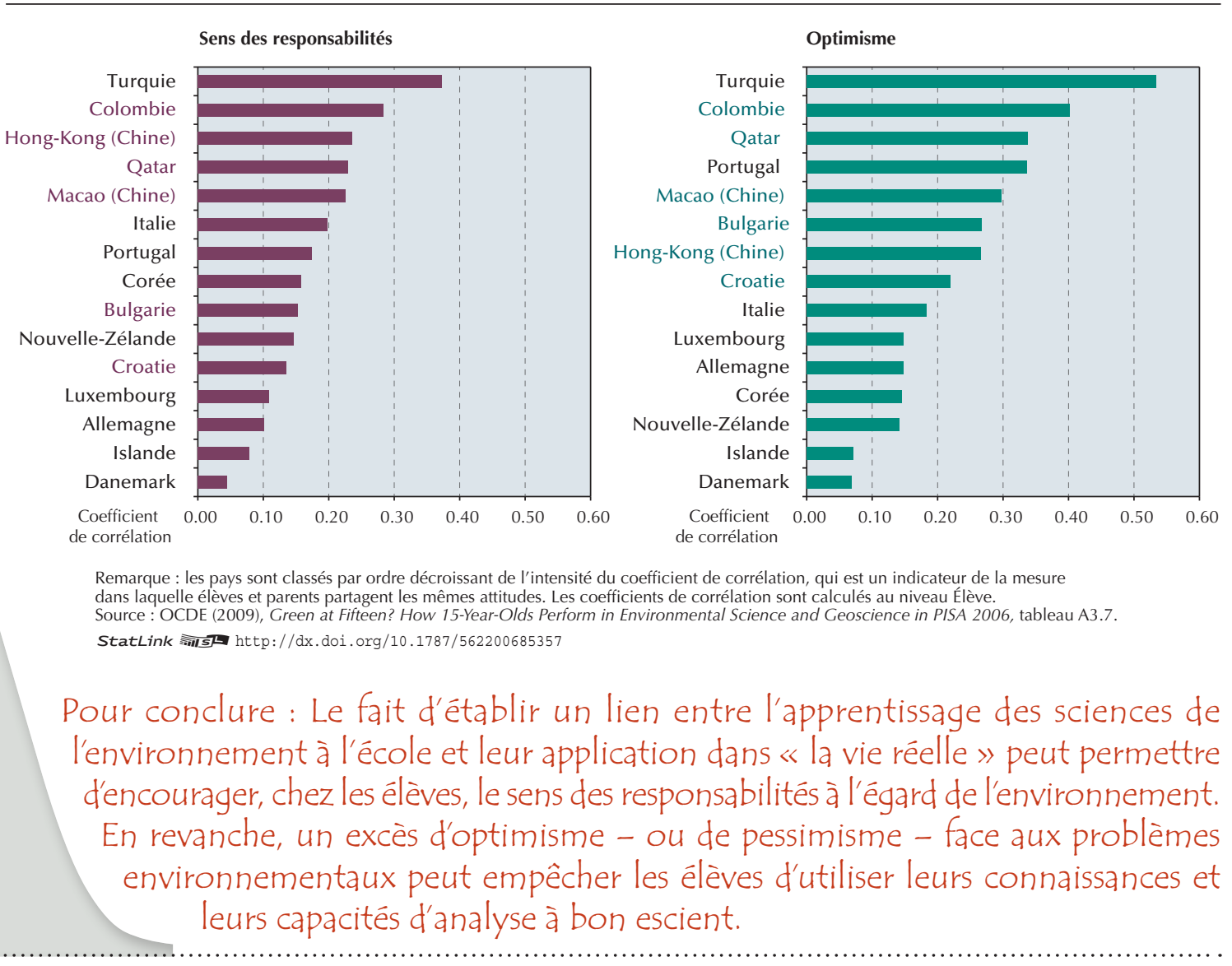

Pour tout complément d'information

Contacter Francesca.Borgonovi (Francesca.Borgonovi@oecd.org)

Consulter Green at Fifteen? How 15-year-olds Perform in Environmental Science and Geoscience in PISA 2006, Éditions OCDE.

\begin{tabular}{ll}
\hline & Prochain numéro \\
Voir & Où en sont les élèves issus de l'immigration \\
www.pisa.oecd.org & dans les établissements d'enseignement \\
www.oecd.org/pisalinfocus & défavorisés?
\end{tabular}

